\title{
Valorisation du surnageant d'effluents de brasserie dans la production de planctons pour la pisciculture : une alternative pour la protection de l'environnement dans les pays du Sud
}

\author{
Mouhamadou Nourou Dine Liady ${ }^{(1)}$, Baruc Kpèhouénou Goussanou ${ }^{(1)}$, Richard Adandé ${ }^{(1)}$, \\ Agossou D. Pacôme Noumavo ${ }^{(2)}$, Louis Amani Kouadio ${ }^{(3)}$, Fouad Zouhir ${ }^{(4)}$, \\ Martin Pépin Aïna ${ }^{(5)}$,Émile Didier Fiogbe ${ }^{(1)}$ \\ (1) Université d'Abomey-Calavi. Faculté des Sciences et Techniques. Laboratoire de Recherche sur les Zones Humide \\ (LRZH). BP 526. Cotonou (Bénin). E-mail : liadynouroudine@gmail.com \\ (2) Université d'Abomey-Calavi. Faculté des Sciences et Techniques. Laboratoire de Microbiologie et des Technologies \\ Alimentaires. Cotonou (Bénin). \\ (3) University of Southern Queensland. Centre for Applied Climate Sciences. West Street. Toowoomba, Queensland \\ (Australia). \\ (4) Université de Liège. Département Sciences et Gestion de l'Environnement. Unité Assainissement et Environnement. \\ Avenue de Longwy, 185. BE-6700 Arlon (Belgique). \\ (5) Université d'Abomey-Calavi. Institut National de l'Eau. Laboratoire des Sciences et Techniques de l'Eau. 01 BP 2009. \\ Cotonou (Bénin).
}

Reçu le 18 mai 2019, accepté le 3 septembre 2020, mis en ligne le 14 septembre 2020.

Cet article est distribué suivant les termes et les conditions de la licence CC-BY (http://creativecommons.org/licenses/by/4.0/ deed.fr)

Description du sujet. Déterminer les conditions optimales d'utilisation des effluents de brasserie en production de phytoplancton.

Objectifs. Déterminer le potentiel nutritif, les conditions d'utilisation et la dépollution réalisée suite à l'utilisation d'effluents de brasserie en aquaculture.

Méthode. Le surnageant a été analysé. Ensuite, cinq traitements ont été ensemencés de Scenedesmus quadricauda et suivis (biomasses et paramètres physico-chimiques) pendant 27 jours. Finalement, les taux d'abattements des charges polluantes initiales ont été déterminés.

Résultats. Le surnageant n'est pas conforme à la norme béninoise, mais présente un potentiel nutritif élevé. La nonneutralisation du pH, initialement acide, occasionne un démarrage tardif de la croissance algale. Le traitement à $10 \%$ présente la production maximale et d'importants abattements de charges polluantes.

Conclusions. Dans un contexte où il n'y a pas de station d'épuration fonctionnelle, il serait intéressant d'envisager un partenariat gagnant-gagnant entre les brasseries et les pisciculteurs. Les conditions optimales d'utilisation incluent une dose optimale et une neutralisation préalable du $\mathrm{pH}$.

Mots-clés. Réutilisation des eaux, aquaculture, pollution, phytoplancton.

Valorisation of the supernatant of brewery effluent in plankton production for fish farming: an alternative for environment protection in southern countries

Description of the subject. Identifying optimal conditions for the use of brewery effluents in phytoplankton production.

Objectives. To determine the nutrient potential of the supernatant of this effluent, conditions for its use in phytoplankton production and the depollution that results from its use.

Methods. Firstly, the supernatant of the effluent was analyzed. Five triplicate treatments were then seeded with Scenedesmus quadricauda, and followed for 27 days through measurements of biomass and physicochemical parameters. Finally, abatement rates of the initial polluting loads were determined. 
Results. Although the supernatant does not comply with the Benin standard, it does have a high nutrient potential. Not neutralizing the acidic initial $\mathrm{pH}$ led to a delay in algal growth. The treatment that contained $10 \%$ supernatant presented the highest algal production and significant reductions in polluting loads.

Conclusions. In contexts where there is no functional wastewater treatment plan, it would be interesting to set up a win-win partnership between breweries and fish farmers. Optimal conditions would include optimal dose and prior pH neutralization.

Keywords. Water reuse, aquaculture, pollution, phytoplankton.

\section{INTRODUCTION}

Malgré les efforts consentis depuis plusieurs années, l'OMS (2015) estime que $32 \%$ de la population mondiale n'a pas accès à l'assainissement. En réalité, ces estimations ne prennent pas en compte les effluents industriels qui sont également mal gérés dans les pays du Sud, alors qu'au regard de leurs caractéristiques, certains d'entre eux, comme ceux des brasseries, peuvent être valorisés dans la production de plancton (Choi, 2016) pour réduire les couts de productions piscicoles. Au Bénin, par exemple, ces couts sont élevés à cause de la cherté des congelés d'artémias importés (Sodjinou et al., 2016) que les pisciculteurs utilisent, à défaut de planctons vivants, plus satisfaisants pour les besoins des premiers stades de développement des poissons (Barnabe, 1976). Aussi, dans des contextes où il n'y a pas de station fonctionnelle de traitement des eaux usées, serait-il intéressant de valoriser ces effluents? À notre connaissance, l'effet de leur utilisation directe sans neutralisation préalable du $\mathrm{pH}$ n'est pas documenté. Ce travail vise à déterminer les potentiels polluants et nutritifs de ces effluents, les conditions optimales pour leur valorisation et l'impact d'une telle valorisation sur la dépollution.

\section{MATÉRIEL ET MÉTHODES}

\subsection{Potentiels polluant et nutritif}

Vingt-sept échantillons bruts de brasserie ont été prélevés conformément à Klingel et al. (2002), de février à avril 2018, puis laissés décanter pendant $48 \mathrm{~h}$ avant de récupérer leurs surnageants. Les paramètres indicateurs de pollution des eaux de brasserie (République du Bénin, 2001) et les macronutriments présents dans le milieu BBM (Bold Basal Medium) ont été analysés dans les surnageants suivant Rodier et al. (2009). Sur la base des informations reçues, la quantité moyenne de surnageants rejetée a été estimée à $5 \mathrm{~m}^{3} \cdot \mathrm{j}^{-1}$ (données non publiées) et les quantités de contaminants rejetées ont pu être calculées.

\subsection{Culture mère de Scenedesmus quadricauda}

La culture mère a été initiée à partir d'une cellule de Scenedesmus quadricauda, isolée suivant Andersen \&
Kawachi (2005), provenant d'un étang situé à la station du Laboratoire de Recherche sur les Zones Humides de l'Université d'Abomey-Calavi (LRZH/UAC) et identifiée à l'aide de la clé de Compère (1976). Elle a été cultivée dans le milieu BBM, sous une agitation de $120 \mathrm{rpm}$, un cycle jour / nuit de $16 \mathrm{~h} / 8 \mathrm{~h}$ et une exposition lumineuse de $146,4 \pm 14,64 \mu \mathrm{mol} \cdot \mathrm{m}^{-2} \cdot \mathrm{s}^{-1}$ à l'aide d'une lampe à sodium haute pression (OSRAM, GmbH, Germany).

\subsection{Cultures expérimentales}

Le test a été réalisé sur cinq triplicats de traitements, constitués de différents taux de surnageant (T0 [0\%], T1 [5\%], T2 [10\%], T3 [20\%] et T4 [40\%]) et d'eau d'un forage situé à la station du LRZH/UAC, dont les caractéristiques ont été prises en compte dans l'analyse de la contribution du système à l'épuration de l'effluent. Chaque traitement a été ensemencé avec une quantité de culture mère (centrifugée à $3000 \mathrm{rpm}$ pendant 15 min et débarrassée du milieu $\mathrm{BBM}$ ) pour obtenir initialement $1.10^{6}$ cellules $\cdot \mathrm{ml}^{-1}$. Les cultures expérimentales ont été réalisées en batch pendant 27 jours, sur des agitateurs magnétiques dans les mêmes conditions que la culture mère.

\subsection{Suivi des cultures}

Les densités algales ont été suivies dans la culture mère par mesure d'absorbance (spectrophotomètre à simple faisceau, HACH DR/2800) à l'aide d'une droite de calibration et, dans les cultures expérimentales, par comptage au microscope à l'aide d'une cellule de Neubauer. Dans les deux cas, les biomasses sèches ont été estimées à partir de conversions des densités algales, à l'aide d'une droite de calibration qui a permis d'estimer à $19,9 \cdot 10^{-12} \mathrm{~g}$ le poids sec moyen d'une cellule de S.quadricauda. La température, le $\mathrm{pH}$ et l'oxygène dissous ont été mesurés à l'aide d'une multisonde HANNA (modèle HI9829).

\subsection{Analyses statistiques et traitement des données}

Les données ont été analysées à l'aide du logiciel STATISTICA (Statsoft.inc.), au seuil de $5 \%$. Le potentiel nutritif a été étudié à l'aide du test $t$ de Student. Les effets des traitements ont été étudiés à l'aide d'une ANOVA. Les comparaisons multiples 
ont été réalisées à l'aide du test HSD de Tukey. La contribution du système à l'épuration de l'effluent a été évaluée à travers des calculs de taux d'abattement des concentrations initiales des paramètres chimiques mesurés.

\section{RÉSULTATS}

\subsection{Potentiels polluant et nutritif}

Le surnageantn'estpasconformeàlanorme (République du Bénin, 2001) concernant la $\operatorname{DBO}_{5}\left(241,15 \mathrm{~kg} \cdot \mathrm{j}^{-1}\right.$ à $\left.48,23 \pm 23,42 \mathrm{~g} \cdot \mathrm{l}^{-1}\right)$, la DCO $\left(1156,85 \mathrm{~kg} \cdot \mathrm{j}^{-1}\right.$ à $231,37 \pm$ $\left.171,24 \mathrm{~g} \cdot \mathrm{l}^{-1}\right)$, les MES $\left(241,15 \mathrm{~kg} \cdot \mathrm{j}^{-1}\right.$ à 48,23 $\left.\pm 23,42 \mathrm{~g} \cdot \mathrm{l}^{-1}\right)$, l'azote Kjeldahl $\left(13,55 \mathrm{~kg} \cdot \mathrm{j}^{-1}\right.$ à $\left.2,71 \pm 1,95 \mathrm{~g} \cdot \mathrm{l}^{-1}\right)$ et le $\mathrm{pH}(4,4 \pm 0,2)$. Mais il est plus riche que le milieu BBM (Tableau 1).

\subsection{Dose optimale du surnageant et contribution de sa valorisation à l'épuration}

On observe un démarrage tardif de la croissance algale dans tous les traitements sauf T0 (Figure 1), des biomasses moyennes significativement plus élevées dans T2 et T1, ainsi qu'une augmentation d'oxygène dissout et de $\mathrm{pH}$ inversement proportionnelle au taux d'effluent présent dans les traitements (Tableau 2). $\mathrm{T} 1$, T2 et T3 ont permis d'obtenir des abattements de l'azote minéral $\left(\mathrm{N}-\mathrm{NH}_{4}^{+}+\mathrm{N}^{+} \mathrm{NO}_{3}{ }^{-}\right)$, des

Tableau 1. Caractéristiques du surnageant de l'effluent de brasserie et du milieu BBM (Bold Basal Medium) - Characteristics of the supernatant of brewery effluent and of BBM (Bold Basal Medium).

\begin{tabular}{|c|c|c|c|}
\hline \multirow[t]{2}{*}{ Paramètre } & \multicolumn{2}{|c|}{ Surnageant de l'effluent de la SOBEBRA } & \multirow[t]{2}{*}{ BBM } \\
\hline & Moyenne & Écart-type & \\
\hline $\operatorname{MES}\left(\mathrm{g} \cdot \mathrm{l}^{-1}\right)$ & 48,23 & 23,42 & 0 \\
\hline $\operatorname{MVS}\left(g \cdot 1^{-1}\right)$ & 43,67 & 23,35 & 0 \\
\hline MVS (\% MES) & 89,28 & 6,56 & 0 \\
\hline $\mathrm{N}-\mathrm{NO}_{3}\left(\mathrm{mg} \cdot \mathrm{l}^{-1}\right)$ & 4,33 & 2,02 & 41,18 \\
\hline $\mathrm{N}-\mathrm{NH}_{3}\left(\mathrm{mg} \cdot \mathrm{l}^{-1}\right)$ & 357,78 & 148,52 & 0 \\
\hline $\mathrm{N}_{\text {org }}\left(\mathrm{mg} \cdot \mathrm{l}^{-1}\right)$ & 2349,56 & 1312,99 & 0 \\
\hline $\mathrm{P}_{\text {org }}\left(\mathrm{mg} \cdot \mathrm{l}^{-1}\right)$ & 121,89 & 45,96 & - \\
\hline $\mathrm{P}-\mathrm{PO}_{4}^{3-}\left(\mathrm{mg} \cdot \mathrm{l}^{-1}\right)$ & 352,13 & 115,30 & 53,25 \\
\hline $\mathrm{N}_{\text {minéral }} / \mathrm{P}_{\text {minéral }}$ & 1,08 & 0,43 & 0,77 \\
\hline $\operatorname{DCO}\left(\mathrm{g} \mathrm{d}^{\prime} \mathrm{O}_{2} \cdot 1^{-1}\right)$ & 231,37 & 114,57 & - \\
\hline $\mathrm{DBO}\left(\mathrm{g} \mathrm{d}^{\prime} \mathrm{O}_{2} \cdot \mathrm{l}^{-1}\right)$ & 7,59 & 2,06 & - \\
\hline $\mathrm{Ca}^{2+}\left(\mathrm{mg} \cdot \mathrm{l}^{-1}\right)$ & 178,67 & 64,18 & 83,8 \\
\hline $\mathrm{Mg}^{2+}\left(\mathrm{mg} \cdot \mathrm{l}^{-1}\right)$ & 153,27 & 58,63 & 7,32 \\
\hline pH (-) & 4,4 & 0,16 & 7 \\
\hline
\end{tabular}




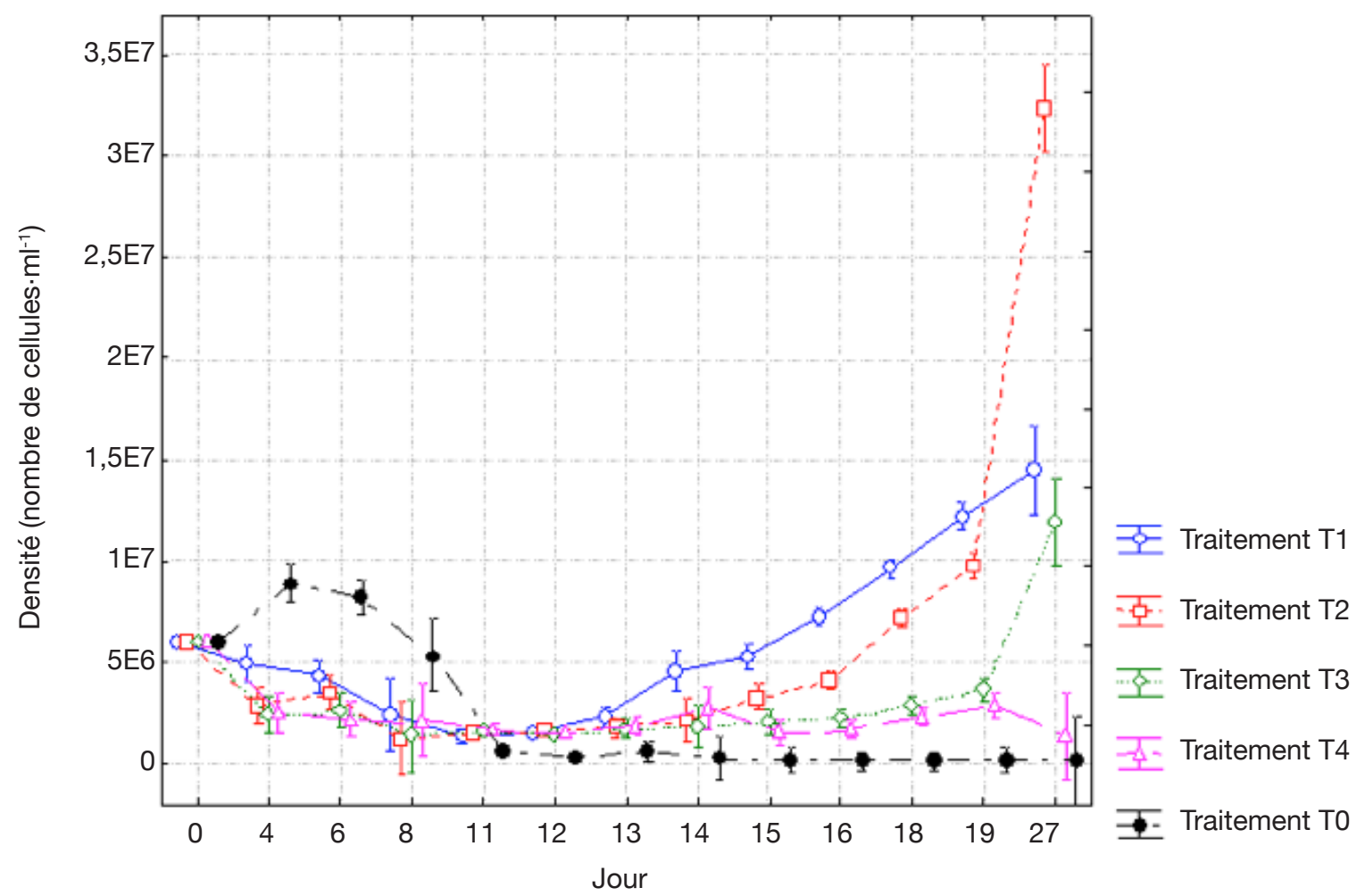

Figure 1.Évolution de la densité de Scenedesmus quadriquada dans les traitements - Evolution of Scenedesmus quadriquada's density in the treatments.

Les barres verticales représentent les intervalles de confiance à $0,95 \%$ - the vertical bars represent the confidence intervals at $0.95 \%$.

phosphates et de la $\mathrm{DBO}_{5}$ respectivement de 68 à $93 \%$, 44 à $55 \%$ et -9 à $65 \%$. T2 domine les autres concernant l'abattement des teneurs en $\mathrm{N}-\mathrm{NO}_{3}^{-}, \mathrm{N}_{-} \mathrm{NH}_{3}$ et $\mathrm{P}_{-} \mathrm{PO}_{4}^{3-}$; par contre, $\mathrm{T} 3$ présente le meilleur abattement de la $\mathrm{DBO}_{5}$.

\section{DISCUSSION}

\subsection{Potentiels polluant et nutritif}

Les potentiels polluants obtenus sont similaires à ceux reportés dans la littérature pour le $\mathrm{pH}$, la DCO, la DBO, les nitrates et les phosphates (Enitan et al., 2015 et les références qui y sont citées). Par contre, ils leur sont différents concernant le ratio DBO/DCO, du fait certainement des modes de constitution des échantillons (Leclercq et al., 1985). La richesse du surnageant, comparativement au milieu BBM, lui confère un potentiel nutritif exploitable en production de planctons.

\subsection{Dose optimale du surnageant et contribution de sa valorisation à la dépollution}

Les abattements de concentrations de polluants observés dans T2 sont du même ordre de grandeur que ceux obtenus par Mata et al. (2012) et par Choi (2016) sur des effluents de brasserie dont le pH a été préalablement neutralisé avec, dans les deux cas, une meilleure production de biomasse. Ces différences pourraient s'expliquer par la différence entre les espèces algales et, surtout, par la différence de $\mathrm{pH}$ initial. Les $\mathrm{pH}$ initiaux acides occasionnent un démarrage tardif de la croissance algale, suivi d'une consommation des bicarbonates qui s'intensifie au fil de l'augmentation de la biomasse, avec pour conséquence une augmentation $\mathrm{du} \mathrm{pH}$ par dissociation de l'acide carbonique régulièrement formé. Ces observations sont en accord avec les conclusions de Xiao et al. (2011) qui ont montré que S. quadricauda s'adapte aux $\mathrm{pH}$ compris entre 6,5 et 8,5 et avec celles de Jacob-Lopes et al. (1987) cités par Xiao et al. (2011), qui montrent que les algues peuvent changer le $\mathrm{pH}$ du milieu de leur culture par leurs métabolismes. Ainsi, les conditions optimales pour la valorisation du surnageant en production de plancton incluent à la fois une dose optimale et une neutralisation préalable du $\mathrm{pH}$.

\footnotetext{
${ }^{1}$ Jacob-Lopes E., Laceira L. M. C. F. \& Franco T. T., 1987. Biochem. Eng. J., 40, 27.
} 


\section{CONCLUSIONS}

Dans un contexte où il n'y a pas de station d'épuration fonctionnelle, il serait intéressant d'envisager un partenariat gagnant-gagnant entre les brasseries et les pisciculteurs. Les conditions optimales d'utilisation incluent une dose optimale et une neutralisation préalable du $\mathrm{pH}$.

\section{Bibliographie}

Andersen R.A. \& Kawachi M., 2005. Traditional isolation techniques. In: Andersen R.A., ed. Algal culturing techniques. London: Elsevier Academic Press, 83-101.

Barnabe G., 1976. Élevage larvaire du loup Dicentrarchus labrax (L.) (Pisces serranidae) à l'aide d'aliment sec composé. Aquaculture, 9, 237-252.

Choi H., 2016. Parametric study of brewery wastewater effluent treatment using Chlorella vulgaris microalgae. Environ. Eng. Res., 21(4), 401-408.

Compère P., 1976. Observations taxonomiques et nomenclaturales sur quelques Desmidites (Chlorophycophyta) de la région du lac Tchad (Afrique centrale). Bull. Jard. Bot. Natl. Belg., 46, 455-470.

Enitan A.M. et al., 2015. Characterization of brewery wastewater composition. Int. J. Environ. Ecol. Eng., 9(9), 1073-1076.

Klingel F., Montangero A., Koné D. \& Strauss M., 2002. Faecal sludge management in developing Countries - A planning manual. Dübendorf, Switzerland: Eawag.
Leclercq S., Jacq V. \& Tine E., 1985. Essais de valorisation de sous-produits de brasserie par fermentation méthanique: procédé «contact anaérobie». Dakar: ORSTOM.

Mata T.M., Melo A.C., Simões M. \& Caetano N.S., 2012. Parametric study of a brewery effluent treatment by microalgae Scenedesmus obliquus. Bioresour. Technol., 107, 151-158, doi.org/10.1016/j.biortech.2011.12.109

OMS, 2015. Rapport sur les progrès accomplis et évaluation des O.M.D: progrès en eau potable et en matière d'assainissement 1990-2015. Genève, Suisse : OMS.

République du Bénin, 2001. Décret n²001-109 du 4 avril 2001, fixant les normes de qualité des eaux résiduaires en république du Bénin.

Rodier J. et al., 2009. L'analyse de l'eau. 9æè éd. Paris : Dunod.

Sodjinou E. et al., 2016. Aliments, ressources alimentaires et pratiques de nourrissage dans les exploitations piscicoles du Sud-Bénin. Porto-Novo : INRAB.

Xiao R., Chen R., Zhang H.-Y. \& Li H., 2011. Microalgae Scenedesmus quadricauda grown in digested wastewater for simultaneous $\mathrm{CO}_{2}$ fixation and nutrient removal. J. Biobased Mater. Bioenergy, 5, 234-240. 